\title{
Spontaneous replication fork collapse regulates telomere length homeostasis in wild type cells
}

\author{
Margherita Paschini ${ }^{1,2, \uparrow}$, Cynthia M. Reyes ${ }^{1,2, \dagger}$, Abigail E. Gillespie ${ }^{1,2}$, Karen A. Lewis ${ }^{4, \ddagger}$, Leslie \\ W. Glustrom ${ }^{4}$, Tatyana O. Sharpee ${ }^{3}$, Deborah S. Wuttke ${ }^{4}$ and Victoria Lundblad ${ }^{1,2, *}$. \\ ${ }^{1}$ Division of Biological Sciences, University of California San Diego, La Jolla, CA 92093-0130, \\ USA. \\ ${ }^{2}$ Molecular and Cellular Biology Laboratory, Salk Institute for Biological Studies, La Jolla, CA \\ 92037-1099, USA. \\ ${ }^{3}$ Computational Neurobiology Laboratory, Salk Institute for Biological Studies, La Jolla, CA \\ 92037-1099, USA. \\ ${ }^{4}$ Department of Biochemistry, University of Colorado, Boulder, Colorado 80309-0596, USA. \\ $\S$ Current address: Stem Cell Program and Divisions of Hematology/Oncology and Pulmonary \& \\ Respiratory Diseases, Boston Children's Hospital, Boston, MA 02115, USA. \\ $\ddagger$ Current address: Department of Chemistry and Biochemistry, Texas State University, San \\ Marcos, TX 78666, USA. \\ * Victoria Lundblad.
}

Email: lundblad@salk.edu

\section{Classification \\ Biological Sciences}

\section{Keywords}

telomerase, telomere, Cdc13, Stn1, t-RPA, replication fork collapse, Rad51.

\section{Author Contributions}

M.P., C.M.R. and A.E.G. performed all of the in vivo experiments; M.P., K.A.L., and L.W.G. analyzed wild type and mutant Cdc13 binding to chromatin or ssDNA substrates; T.O.S. performed statistical analysis; D.S.W. and V.L. consulted extensively during the project and V.L. wrote the manuscript. 


\begin{abstract}
Telomeres present unique challenges for genomes with linear chromosomes, including the inability of the semi-conservative DNA replication machinery to fully duplicate the ends of linear molecules. This is solved in virtually all eukaryotes by the enzyme telomerase, through the addition of telomeric repeats onto chromosome ends. It is widely assumed that the primary site of action for telomerase is the single-stranded G-rich overhang at the ends of chromosomes, formed after DNA replication is complete. We show here that the preferred substrate for telomerase in wild type yeast is instead a collapsed fork generated during replication of duplex telomeric DNA. Furthermore, newly collapsed forks are extensively elongated by telomerase by as much as $\sim 200$ nucleotides in a single cell division, indicating that a major source of newly synthesized telomeric repeats in wild type cells occurs at collapsed forks. Fork collapse and the subsequent response by telomerase are coordinated by the dual activities of a telomerededicated RPA-like complex, which facilitates replication of duplex telomeric DNA and also recruits telomerase to the fork, thereby ensuring a high probability of re-elongation if DNA replication fails. We further show that the ability of telomerase to elongate newly collapsed forks is dependent on the Rad51 protein, indicating that telomerase activity in response to fork collapse proceeds through a regulatory pathway distinct from how telomerase engages fully replicated chromosome termini. We propose a new model in which spontaneous replication fork collapse and the subsequent response by telomerase is a major determinant of telomere length homeostasis.
\end{abstract}




\section{Introduction}

Telomeres - the natural ends of linear eukaryotic chromosomes - play dual roles in ensuring genome stability and dictating long-term proliferation of cells. In cells that rely on continuous proliferation, telomeres must overcome the DNA end replication problem, which stems from the inability of the semi-conservative DNA replication machinery to fully replicate the ends of linear molecules. In addition, chromosome termini must be shielded from DNA damage signaling pathways and subsequent inappropriate DNA repair, a process that is often referred to as chromosome end protection. These two threats to chromosome integrity are addressed by telomere-dedicated factors that associate with either duplex telomeric DNA or the single-stranded G-rich extension present at chromosome ends, aided by sequence-specific recognition of the Grich telomeric repeats that characterize chromosome termini in most species (1-3).

Central to these activities is the enzyme telomerase, which solves the end-replication problem and provides the sequence-specific platform for telomere-binding proteins, through the templated addition of telomeric repeats to chromosome ends (4). Failure to maintain this telomeric tract results in an eventual block to cell division; in telomerase-expressing cells, therefore, there is a considerable regulatory investment to ensure that telomere length is stably maintained, through species-specific activities as well as conserved pathways (reviewed in 2, 3). One such mechanism is enhancement of enzyme activity by telomerase-associated factors, which has been observed in several disparate species, suggesting that this may be a widely conserved regulatory step. In human cells, this is promoted by the TPP1-POT1 complex, which increases the activity and processivity of telomerase (5) through an interaction between a surface on TPP1 and the Nterminal domain of the catalytic subunit of telomerase (6). In budding yeast, the Est3 subunit of budding yeast telomerase, which exhibits a striking structural similarly to a domain of human TPP1 (7) and binds the comparable N-terminal domain of the yeast catalytic subunit (8), also appears to enhance enzyme activity $(9,10)$. Telomerase is also actively recruited to chromosome termini through interactions with telomere-bound proteins, although divergent mechanisms are employed in different species. In humans, recruitment is mediated by binding of 
the TPP1 shelterin subunit to the catalytic subunit of telomerase $(11,12)$, although other shelterin proteins also contribute (reviewed in 3). In contrast, telomerase recruitment in budding yeast relies on an interaction between a regulatory subunit of telomerase (Est1) and the telomerebound Cdc13 protein (13-15); disruption of this interaction leads to a phenotype comparable to that of a telomerase-null strain (16). Cdc13 performs its role at telomeres in association with two other proteins (Stn1 and Ten1) as a heterotrimeric complex with striking structural and architectural similarities to the canonical RPA complex $(17,18)$. Specificity for chromosome ends is mediated by a high affinity DNA binding domain in Cdc13, which exhibits exceptional sequence preference for single-stranded G-rich telomeric sequences in budding yeast (19) and closely related species (20), leading us to refer to it as a t-RPA complex. This contrasts with the genome-wide role performed by this conserved RPA-like complex in multiple other species (2124), consistent with the lack of strong specificity for telomeric DNA (25).

Despite several layers of positive regulation, telomerase nevertheless elongates only a subset of telomeres in each cell division, with a bias for shorter telomeres in both yeast and human cells (26-28). A long-standing model (29) has proposed that this restricted activity is the result of a cisacting process that dictates the accessibility of individual telomeres to telomerase. Mechanistically, this is thought to be accomplished by a feedback mechanism that senses the length of each telomere, by "counting" the number of proteins bound to the duplex telomeric tract, referred to as the protein counting model (reviewed in 30,31 ); this information subsequently determines whether a telomere is in a telomerase-extendible or telomerase-non-extendible state. This would promote preferential elongation of shorter-than-average telomeres by telomerase, due to the increased probability that shorter telomeres will persist in an "extendible" state, while restricting telomerase activity at longer-than-average telomeres (27), thereby ensuring that telomeres are tightly maintained around an average length. In particular, telomeres short enough to trigger a block to cell cycle progression are assumed to be transient, as they would be rapidly targeted for re-elongation. However, the process by which information from this length-sensing process is relayed to telomerase, as well as the mechanism responsible for switching telomeres 
between telomerase-extendible vs. telomerase-non-extendible states, have remained elusive (31). In addition, methods that have monitored the length of individual telomeres in both yeast and human cells (32-35) have revealed that a subset of individual chromosome termini exhibit substantial deviations from the average length in telomerase-plus cells.

The above model (as well as essentially every model in telomere biology) also assumes that the primary substrate for telomerase is the single-stranded G-rich overhang present at the ends of chromosomes, produced after passage of the replication fork through duplex telomeric DNA (4, $36,37)$. However, in cells undergoing high levels of replication stress, broken ends created by replication fork collapse can be elongated by telomerase (38-40). Left unaddressed by these prior studies is whether this was a pathological response to replication stress or instead reflected a physiological property of telomerase that was employed by unstressed wild type cells. To evaluate this, we developed an assay to monitor fork collapse, and the subsequent response by telomerase, during replication of duplex telomeric DNA. By monitoring the molecular footprint of telomerase activity in the same cell division in which fork collapse occurs, we show that termini generated by spontaneous fork collapse in wild type cells are elongated far more frequently than fully replicated telomeres. In addition, telomerase-mediated elongation of a newly collapsed fork in a single cell division is quite extensive, in contrast to the more limited telomerase activity observed at fully replicated yeast chromosome ends, indicating that a major source of newly synthesized telomeric repeats occurs at collapsed forks. Furthermore, reducing the ability of telomerase to elongate a newly collapsed fork substantially increased the sub-population of shorter-than-average telomeres, with a resulting impact on overall telomere length. Collectively, these observations argue that the choice between telomerase-extendible vs. telomerase-nonextensible states (27) is instead a choice between two temporally and structurally distinct substrates for telomerase. We propose a new model for telomere length regulation, in which the disparate response of telomerase to two temporally and structurally distinct substrates is a major determinant of telomere length homeostasis. 


\section{Results}

Wild type telomeres exhibit extensive length variation due to founder sequence loss. In telomerase-proficient wild type yeast, numerous studies have shown that the average length of the duplex telomeric tract is remarkably stable. These observations, which have been largely based on low resolution analysis (i.e. Southern blotting), have contributed to the premise that yeast telomeres are maintained around a fixed average length. However, PCR-based assessments of wild type telomere length at single-nucleotide resolution have shown that individual chromosome ends exhibit extensive length variation, including a sub-population of markedly shorter telomeres $(34,35,41)$. We used a modified version of this PCR-based protocol to conduct an expanded analysis of wild type Chr VI-R and Chr I-L telomere length; modifications included (i) elimination of a size selection step to ensure that very long and very short subpopulations were not excluded and (ii) a substantial increase in the number of isolates in order to obtain a reproducible assessment of the extent of telomere length variation (see Materials and Methods for details). Analysis of 262 and 240 independent isolates of Chr VI-R and Chr I-L telomeres, respectively, showed that the lengths of individual termini spanned more than $450 \mathrm{bp}$ (Fig. $1 A$ and $1 B$; SI Appendix, Tables S1 and S2), with 28.2\% and 20\%, respectively, diverging from the mean (either shorter or longer) by more than $100 \mathrm{bp}$. An independent repeat yielded a virtually identical telomere length profile for Chr I-L (Fig. 1C), arguing that this extensive length variation was not due to stochastic variation and instead reflected an inherent feature of wild type telomere length homeostasis.

These data were further analyzed by re-aligning the sequenced Chr VI-R and Chr I-L telomeres shown in Fig. $1 A$ and $1 B$ based on the length of founder $G_{1-3} T D N A$ inherited by each isolate (Fig. $1 D$ and $1 E$ ); the minimal length of the $\mathrm{G}_{1-3} \mathrm{~T}$ DNA present in the Chr VI-R or Chr I-L chromosome that founded each colony was determined as described in Material and Methods and SI Appendix, Fig. S1. The average rate of founder sequence loss was 3.9 bp per cell division for both Chr VI-R and Chr I-L telomeres (calculated as described in Material and Methods), which is consistent with previous estimates for an average erosion rate of 3-5 nucleotides/cell division (42, 
43). Sequence loss from chromosome ends has generally been assumed to be due to terminusspecific processes. However, for a subset of Chr VI-R and Chr I-L telomeres, founder sequence loss was substantially greater, as $18.7 \%$ of $\mathrm{Chr}$ VI-R telomeres and $9.1 \%$ of $\mathrm{Chr} \mathrm{I}-\mathrm{L}$ telomeres inherited less than $125 \mathrm{bp}$ of founder DNA during the $\sim 28$ generations of growth prior to DNA isolation (Fig. 1D and 1E). For this sub-population, this exceeds the sequence loss predicted by terminus-specific erosion by more than $100 \mathrm{bp}$, arguing that additional activity(s) contribute to loss of founder sequence DNA in wild type cells. As suggested previously $(34,44,45)$, one mechanism could be chromosome breakage due to fork collapse during replication of duplex telomeric DNA.

Notably, when images of total length profiles were superimposed on the corresponding Chr VI-R or I-L founder length profiles in Fig. $1 D$ and $1 E$, this revealed a striking relationship. Although founder vs. total length profiles were offset by an average of 43 and $37 \mathrm{bp}$ for Chr VI-R and Chr I$\mathrm{L}$, respectively, the total length profile was remarkably similar to the founder length profile for both telomeres. This argues that founder length dictates overall telomere length, even in telomeraseproficient cells. If so, mechanism(s) that drive loss of founder sequence DNA, including erosionindependent processes such as replication fork collapse, should be critical determinants of telomere length homeostasis. A major goal of this study was to assess the contribution of fork collapse at telomeres in wild type cells.

Spontaneous replication fork collapse at an interstitial telomeric tract. Although past research has shown that fork collapse in response to high levels of replication stress can impact telomere function, it has been unclear whether this contributes to telomere length homeostasis in unstressed wild type cells. As a first step in evaluating this, we developed an assay to measure spontaneous fork collapse (Fig. 2A; SI Appendix, Fig. S3), by inserting 390 bp of $\mathrm{G}_{1-3} \mathrm{~T}$ telomeric DNA at a site on Chr IX that was $27 \mathrm{~kb}$ from the natural terminus and immediately adjacent to ARS922, a high efficiency origin of replication (46). A key feature of this assay was that the distal $27 \mathrm{~kb}$ portion of Chr IX was dispensable for viability; as a result, fork collapse events could be 
recovered as viable cells. This $27 \mathrm{~kb}$ segment was also marked by the ADE2 gene (with a corresponding ade2- $\Delta$ deletion at the endogenous locus), which allowed visual identification of cell divisions in which fork collapse occurred as red $\mathrm{Ade}^{-}$half-sectors (Fig. 2B). Confirmation that the $\mathrm{Ade}^{-}$phenotype was due to loss of the distal $27 \mathrm{~kb}$ portion of Chr IX employed Southern blot analysis, as well as PCR amplification and sequence analysis of the newly exposed terminus (SI Appendix, Fig. S3), which demonstrated that the interstitial $\mathrm{G}_{1-3} \mathrm{~T}$ tract had become terminal in $100 \%$ of $\geq 100 \mathrm{Ade}^{-}$colonies. This was further supported by monitoring of a second genetic marker (HIS3) downstream from the interstitial telomeric tract, which showed that $100 \%$ of $\sim 250$ $\mathrm{Ade}^{-}$half-sectors were also $\mathrm{His}^{-}$. As described in more detail below, sequence analysis of progeny in the resulting $\mathrm{Ade}^{-}$half-sector could be used to infer the molecular structure of newly formed termini immediately following fork collapse. This has provided a powerful assay for investigating the contribution of spontaneous fork collapse during replication of duplex telomeric DNA in wild type cells.

Two observations support the conclusion that loss of the distal portion of Chr IX was a direct consequence of DNA replication failure. First, the frequency of $\mathrm{Ade}^{-}$sectors was sensitive to conditions that lead to irreversible fork stalling. In a wild type strain, previous work has shown that exposure to even high levels of $\mathrm{HU}$ results in reversible replication fork stalling; cells arrest but resume cell cycle progression once HU is depleted. In contrast, in the S-phase-checkpointdeficient rad53-21 strain, stalled replication forks in response to HU cannot be reversed $(47,48)$. We therefore compared the frequency of $\mathrm{Ade}^{-}$sectors in wild type vs. rad53-21 strains in the presence of $\mathrm{HU}$. In a rad53-21 strain, replication fork collapse at the interstitial telomeric tract was elevated 3-fold when cells were grown in the presence of just $1 \mathrm{mM} \mathrm{HU}$; in the absence of $\mathrm{HU}$, the rad53-21 defect had no effect (Fig. 2C). In contrast, the frequency of Ade ${ }^{-}$sectors in a wild type strain was unaffected by $\mathrm{HU}$ concentrations as high as $40 \mathrm{mM}$. This rad53-21 dependency provided direct evidence that loss of the distal tract occurred in response to replication fork collapse, rather than spontaneous DSB formation. Furthermore, loss of the distal tract was responsive to defects in the replisome itself. When cells from a strain expressing a 
temperature sensitive mutation in DNA pol $\alpha(p o / 1-1)$ were incubated on plates that had been pre-warmed to $23^{\circ}, 30^{\circ}$ or $31^{\circ}$, there was a marked temperature-dependent increase in the frequency of $\mathrm{Ade}^{-}$sectors in pol1-1 colonies, with a 5-fold increase at the semi-permissive temperature of $31^{\circ}$ (Fig. 2D). We note that the instability of this interstitial telomeric tract in response to impaired DNA pol $\alpha$ function is reminiscent of the aphidicolin-induced fragility previously observed at human duplex telomeric repeats (49).

These newly exposed termini were robust substrates for telomerase, as described in the next section. However, recovery of $\mathrm{Ade}^{-}$cells was not dependent on elongation of the newly exposed telomeric tract by telomerase, as the frequency of $\mathrm{Ade}^{-}$half-sectors was unaffected in a telomerase-negative strain bearing a deletion of the telomerase RNA gene TLC1 (Fig. 2E). The senescence phenotype of $\operatorname{Ade}^{-}$t/c1- $\Delta$ half-sectors was also indistinguishable from that of the corresponding $\mathrm{Ade}^{+}$half of each $t / c 1-\Delta$ colony (SI Appendix, Fig. S3), indicating that these newly created termini were not perceived as unrepaired DNA damage but instead exhibited the same characteristics as natural eroding chromosome ends. Moreover, these termini were not subject to telomerase-independent mechanisms such as break-induced replication or sister chromatid recombination, as revealed by examining the molecular structure of newly exposed ends in 12 Ade $^{-}$t/c1- $\Delta$ half-sectors. If the newly collapsed fork was acted upon by non-telomerase-mediated sequence addition mechanisms, the sequence of these newly exposed telomeres should diverge from that of the interstitial tract. Instead, sequence analysis of telomeres recovered from the progeny of each $\operatorname{Ade}^{-}$t/c1- $\Delta$ half-sector showed that in the absence of telomerase, collapsed replication forks were not substrates for either break-induced replication or recombination (Fig. $2 F$ and SI Appendix, Fig. S4).

\section{A collapsed replication fork generates a single terminus that is extensively elongated by}

telomerase. In telomerase-proficient (i.e. wild type) cells, molecular analysis showed that fork collapse at the interstitial tract created a substrate that was readily elongated by telomerase. Due to the slightly degenerate telomeric repeat synthesized by yeast telomerase (42), alignments of 
multiple sequenced telomeres from the progeny of each $\mathrm{Ade}^{-}$half-sector precisely identified the position at which telomerase initiated synthesis, as the point at which the sequence of the newly elongated DNA end diverged from that of the interstitial tract. This was illustrated by transiently expressing a modified telomerase that synthesized an altered telomeric repeat (50) just prior to formation of $\mathrm{Ade}^{-}$half-sectored colonies. In the example shown in Fig $3 A$, analysis of sequenced telomeres from an $\mathrm{Ade}^{-}$half-sector from this experiment revealed that the interstitial tract was extended by mutant telomeric repeats starting at nucleotide 163 , following loss of the distal $27 \mathrm{~kb}$ segment; additional $\mathrm{Ade}^{-}$half-sectors from this experiment are shown in SI Appendix, Fig. S5. In the example shown in Fig. 3B, similar analysis of an $\mathrm{Ade}^{-}$half-sector from a wild type strain showed that telomerase initiated synthesis at nucleotide 57 , following fork collapse at the interstitial telomeric tract.

This analysis also distinguished between whether fork collapse generated a single terminus that was recognized by telomerase vs. two termini that were each independently elongated (see $S I$ Appendix, Fig. $\mathrm{S} 6$ for a schematic illustrating these two possibilities). In the examples shown in Fig. $3 A$ and $3 B$, a common tract of newly synthesized telomeric DNA (indicated by white brackets) was inherited by the majority of $\mathrm{Ade}^{-}$progeny. This pattern of inheritance indicated that in the founder cell division that established each of these two $\mathrm{Ade}^{-}$sectors, fork collapse gave rise to a single 3' terminus that was elongated in the same cell division by telomerase. To assess how frequently this occurred, telomeres from $38 \mathrm{Ade}^{-}$sectors from a wild type yeast strain were analyzed. This identified 20 sectors in which progeny descended from a single telomerase-elongated founder chromosome, similar to the example in Fig. 3B (see SI Appendix, Fig. S7 for additional examples from this category of $\mathrm{Ade}^{-}$half-sectors). These results show that telomerase recognizes and elongates $>50 \%$ of collapsed replication forks, which is far more efficient than the $7 \%$ frequency that has been estimated for telomerase activity at fully replicated telomeres (27). This suggests that the preferred substrate for telomerase in wild type cells is a collapsed fork generated during replication of duplex telomeric DNA. 
The point at which telomerase initiated synthesis for these $20 \mathrm{Ade}^{-}$sectors spanned from 24 to 253 nucleotides across the 390 bp interstitial tract, as indicated by the blue arrows in Fig. 3 C. Telomerase-mediated elongation in a single cell division was also quite extensive for many of these $20 \mathrm{Ade}^{-}$half-sectors. In the example shown in Fig. 3B, 168 nucleotides were synthesized by telomerase in this founder $\mathrm{Ade}^{-}$cell division, based on the sequence inherited by the majority of progeny. For 15 of the $20 \mathrm{Ade}^{-}$half-sectors, newly collapsed forks were elongated by as much as 95 to 215 nucleotides in a single cell division (Fig. 3D and SI Appendix, Fig. S8); this argues that a major source of newly synthesized telomeric repeats by telomerase in wild type cells occurs at collapsed forks. Moreover, the extent of elongation was not determined by the location of the fork collapse, as fork collapse events that occurred at more distal positions in the interstitial tract (150 - $200 \mathrm{bp}$ from the sub-telomeric boundary) were elongated by the same amount (or even more) than a subset of events that occurred in the first $50 \mathrm{bp}$ of the internal tract (Fig. 3D and SI Appendix, Fig. S8). This argues that the extent of telomerase elongation of newly collapsed forks was not regulated in cis by the length of the corresponding duplex $\mathrm{G}_{1-3} T$ tract.

In the remaining $18 \mathrm{Ade}^{-}$half-sectors, alignments of telomeres from progeny did not reveal a pattern consistent with a single founder chromosome that was elongated by telomerase. Instead, individual termini of progeny from each of these 18 sectors exhibited multiple points of sequence divergence, when compared to the interstitial tract, indicating that these newly exposed Chr IX termini were not extended by telomerase until later cell divisions. The approximate positions of fork collapse for the $18 \mathrm{Ade}^{-}$sectors in this category are depicted by red arrows in Fig. $3 \mathrm{C}$; alignments of sequenced telomeres from progeny for a subset of these 18 sectors are shown in SI Appendix, Fig. S9.

To ask if the above observations extended to native telomeres, we also tracked the pattern of addition of mutant telomeric repeats at the natural Chr I-L telomere. To do so, the mutant telomerase RNA described above was transiently expressed in an otherwise wild type strain just prior to plating for single colonies, and progeny from five colonies were examined for 
incorporation of mutant telomeric repeats at the Chr I-L terminus. Colonies were selected without any bias (i.e. without a visual read-out indicating that fork collapse had occurred), which differentiates this experiment from the analysis of $\mathrm{Ade}^{-}$half-sectors described above. $\ln 3$ of the 5 colonies, sequence analysis of Chr I-L telomeres identified progeny telomeres that displayed a substantial loss of founder sequence DNA (192, 196 or 255 nucleotides), coupled with extensive addition (132, 195 or 250 nucleotides) of mutant telomeric repeats (Fig. 3E). These results suggest that spontaneous fork collapse, followed by robust elongation by telomerase, occurs at native telomeres at a readily detectable frequency.

The t-RPA complex facilitates replication of duplex telomeric DNA. Analysis of the $38 \mathrm{Ade}^{-}$ sectors indicated that elongation of newly collapsed forks by telomerase in the first cell division (blue arrows in Fig. $3 C$ ) exhibited an apparent gradient across the interstial tract (Fig. 3F). This bias is consistent with a key prediction of a recent model (31) proposing that telomerase travels with the replication fork, with association decreasing as the fork proceeds towards the terminus. This model further predicts that a telomere-dedicated RPA-like complex (t-RPA) which mediates telomerase recruitment (13-15), also interacts with the replication fork, presumably by binding single-stranded $\mathrm{G}_{1-3} \mathrm{~T}$ DNA that becomes exposed as the fork traverses duplex telomeric DNA. Consistent with this expectation, the Cdc13 subunit of the yeast t-RPA complex bound the interstitial telomeric tract on Chr IX, as measured by chromatin immunoprecipitation, which paralleled its ability to bind $\mathrm{G}_{1-3} \mathrm{~T}$ ssDNA embedded in a duplex DNA substrate in vitro ( $\mathrm{SI}$ Appendix, Fig. S10). Furthermore, a mutation in CDC13 (cdc13-F539A) that reduced the ability of the t-RPA complex to associate with telomeres had a substantial effect on replication fork collapse. This mutation, located in a DNA contact residue on the surface of the high affinity DNAbinding domain of Cdc13 (19), reduced DNA binding in vitro as well as association of Cdc13 with telomeric chromatin (ref; SI Appendix, Fig. S10). Notably, the frequency of fork collapse at the interstitial telomeric tract was increased by more than 10-fold by the cdc13-F539A mutation (Fig. $4 A$ ). This suggests that the yeast t-RPA complex facilitates duplex telomeric DNA replication, 
which parallels observations in other species showing that this conserved RPA-like complex facilitates lagging strand DNA synthesis $(21-23,51,52)$.

We also asked if the ability of telomerase to elongate newly collapsed replication forks was mediated by the telomerase recruitment activity of the t-RPA complex. To test this, newly collapsed forks were examined in an est1-60 mutant strain, which disrupts the recruitmentdependent interaction between Cdc13 and telomerase (15), by analyzing sequence addition in 20 est1-60 Ade $^{-}$half-sectors. In all 20 isolates, elongation of newly collapsed forks by telomerase was eliminated, as shown by analysis of progeny from each est1-60 $\mathrm{Ade}^{-}$half-sector (Fig. 4B; additional examples in SI Appendix, Fig. S11). This genetic result complements an earlier observation showing high levels of recruitment of telomerase to sites of defective DNA replication in fission yeast (39). Consistent with Greider's proposed model (31), these data argue that recruitment of telomerase to the replication fork is mediated by the t-RPA complex, thereby ensuring a high frequency of re-elongation when duplex telomeric DNA replication fails.

\section{Replication fork collapse increases the extent of telomere length variation. If replication}

fork collapse is responsible for the extensive loss of founder sequence DNA observed for a subset of wild type telomeres, as described in Fig. 1, this predicts that an increased frequency of fork collapse should accelerate founder sequence loss, with a corresponding increase in the subpopulation of shorter-than-average telomeres. At the same time, if fork collapse creates a preferred substrate for telomerase at native telomeres similar to the observations at the interstitial telomeric tract (Fig. 3), an increase in fork collapse should expand the sub-population of overelongated telomeres. If these two predictions are correct, altering the frequency of replication fork collapse should increase both of these aspects of telomere length heterogeneity.

To test this, telomere length in the cdc13-F539A strain was assessed using the high resolution assay described in Fig. 1. Analysis of 251 independent cdc13-F539A isolates of Chr I-L recovered after $\sim 28$ generations of growth from single colonies (SI Appendix, Table S3) showed that average telomere length in the $c d c 13-F 539 A$ strain was essentially indistinguishable from 
that of wild type (301 vs. $311 \mathrm{bp}$, respectively). However, the extent of telomere length variation was greatly elevated, as shown by an alignment of the 251 sequenced cdc13-F539A Chr I-L telomeres based on length (Fig. 4C). When this cdc13-F539A telomere length profile was compared to the wild type Chr I-L profile (superimposed in black in Fig. 4C), this revealed a marked increase in sub-populations of much shorter or longer telomeres: $19.5 \%$ of cdc13-F539A termini were $\leq 125$ bp vs. $5.4 \%$ of wild type telomeres $(p<0.0001)$, and $12.4 \%$ were $\geq 475$ bp vs. $1.7 \%$ for wild type $(p<0.0001)$. As we show below, this was due to the combined effects of (i) an increased loss of founder sequence DNA and (ii) an increased frequency of elongation by telomerase. We propose that both occur in response to replication fork collapse.

The impact on founder sequence loss was evaluated by re-aligning the $251 \mathrm{cdc} 13-\mathrm{F} 539 \mathrm{~A}$ telomeres based on the length of founder sequence inherited by each isolate (Fig. 4D). When compared to an image of the wild type founder sequence profile (superimposed in aqua in Fig. $4 D$ ), this showed that founder sequence loss was substantially increased in the cdc13-F539A strain, with the average rate of $c d c 13-\mathrm{F} 539 \mathrm{~A}$ founder sequence loss increased to $7.2 \mathrm{bp} /$ cell division (a 1.8-fold increase relative wild type; Fig. 4E). The effect was particularly pronounced when comparing sub-populations of the shortest telomeres, as the fraction of telomeres that retained $\leq 125 \mathrm{bp}$ of founder DNA increased from $9.2 \%$ in wild type to $31.5 \%$ in the $c d c 13-\mathrm{F} 539 \mathrm{~A}$ strain $(p<0.0001)$. Since the cdc13-F539A strain does not have a resection defect (19), this argues that the increased loss of founder sequence DNA was the consequence of an elevated frequency of fork collapse, rather than increased terminus-specific erosion.

The elevated frequency in fork collapse in the cdc13-F539A strain also increased the extent of telomerase elongation at native telomeres, consistent with the behavior of telomerase in response to fork collapse at the interstitial tract. This was evaluated by comparing the length of DNA synthesized by telomerase at individual Chr I-L telomeres in the cdc13-F539A strain with the extent of synthesis at wild type Chr I-L telomeres (Fig. 4F and $4 G$ ). Although these two data sets represent the accumulation of multiple telomerase-mediated events over $\sim 28$ generations of 
growth for each strain, this comparison nevertheless indicated that telomerase-mediated synthesis was substantially increased in the cdc13-F539A strain. A calculation of the average length synthesized by telomerase per chromosome end showed that Chr I-L telomeres were elongated by an average of $56 \mathrm{bp}$ in the mutant strain, whereas wild type termini were elongated by an average of $27 \mathrm{bp}$. Notably, this increased synthesis by telomerase in the cdc13-F539A strain was asymmetrically distributed, as $7.6 \%$ of telomeres were elongated by $>150 \mathrm{bp}$ in the mutant strain, compared to $1.7 \%$ in wild type (Fig. $4 H$ ). These observations are consistent with the premise that there are two categories of telomerase substrates - one created by fork collapse and a second produced following completion of DNA replication - which are elongated to differing extents. We propose that the increase in the sub-population of over-elongated telomeres in the cdc13-F539A strain was a direct consequence of an increase in one of these two telomerase substrates, as the direct result of an elevated frequency of fork collapse.

As a further test of this premise, we asked if the increased frequency of fork collapse in the cdc13-F539A strain would be suppressed by an exo1- $\Delta$ mutation, and if so, would there be a cosuppressing impact on telomere length homeostasis. Exo1 has been shown to generate ssDNA gaps at perturbed replication forks (53-55); furthermore, loss of Exo1 function suppressed replication fork breakdown in other genetic scenarios (56). Notably, the increased frequency of fork collapse observed in the cdc13-F539A strain was reduced 4.4-fold by an exo1- $\Delta$ mutation (Fig. 4A). In parallel, the telomere length variation exhibited by the cdc13-F539A strain was partially suppressed, as shown by an analysis of 189 independent isolates of the Chr I-L terminus from the cdc13-F539A exo1- $\Delta$ strain (SI Appendix, Table S4). This showed that in the double mutant strain, the average rate of founder sequence loss declined to $5.2 \mathrm{bp} /$ cell division (Fig. $4 E$ and $4 G$ ). In addition, the extent of telomerase elongation of individual telomeres was also partially suppressed (Fig. 4J), with the proportion of telomeres that were extensively elongated by telomerase (> $150 \mathrm{bp}$ ) reduced to a level in the cdc13-F539A exo1- $\Delta$ strain (3.3\%) that was intermediate between the wild type and cdc13-F539A strains (Fig. 4H). Co-suppression of these three $c d c 13-F 539$ A phenotypes - the frequency of fork collapse, the rate of founder sequence 
loss and the extent of telomerase-mediated elongation - by an exo1- $\Delta$ mutation provides additional support for the premise that telomere length homeostasis is regulated by replication fork collapse. In parallel, the pronounced cdc13-F539A growth defect was partially reversed by an exo1- $\Delta$ mutation (Fig. $4 K$ ), suggesting that the essential function of the t-RPA complex is to prevent replication fork collapse. This Exo1-dependent suppression of fork collapse was not specific to the $c d c 13-F 539 A$ mutation, as a $c d c 13$-ts strain (57) also exhibited a substantial increase in fork collapse at a semi-permissive temperature that was similarly suppressed by an exo1- $\Delta$ mutation (Fig. $4 L$ and $4 M$ ).

Telomerase-mediated elongation of newly collapsed forks is mediated by Rad51. A role for fork collapse at telomeres also predicts that if the ability of telomerase to elongate the substrate created by a collapsed fork is impaired, telomere length at native chromosome ends will be altered. An insight into testing this prediction came from the observations showing that fork collapse at the interstitial telomeric tract generated a single terminus that was elongated by telomerase (Fig. 3 and SI Appendix, Fig. S7). One widely discussed mechanism for generating a telomerase substrate $(39,40)$ is fork regression, whereby newly synthesized strands re-anneal to form a four-pronged structure (58). As illustrated in Fig. 5A, fork regression in response to stalled replication of duplex telomeric DNA will produce a single 3' G-rich extension that can be elongated by telomerase, with the resulting progeny descending from a single telomeraseelongated founder chromosome. This pattern of inheritance was observed for more than $50 \%$ of the fork collapse events at the interstitial tract (Fig. $3 C$ ), suggesting that the substrate elongated by telomerase at the interstitial tract is a regressed fork. If so, activities that mediate the remodeling of stalled replication forks into regressed fork structures should affect the ability of telomerase to recognize and/or elongate this category of substrates.

To test this, the pattern of telomerase elongation following fork collapse at the interstitial tract was examined in a rad51- $\Delta$ yeast strain, as Rad51 has been shown to associate with stalled forks to prevent their degradation, as well as facilitating fork regression, in both human and fission yeast 
cells (59-63). When progeny from 28 Ade $^{-}$half-sectors recovered from a rad51- $\Delta$ strain were analyzed, this revealed a striking reduction in sectors in which telomerase elongated a newly collapsed replication fork, from $>50 \%$ in wild type (Fig. $3 C$ ) to $13 \%$ in the rad51- $\Delta$ strain (Fig. $5 B$ and $5 \mathrm{C}$ ). This was accompanied by a notable reduction in the recovery of Ade ${ }^{-}$sectors in the first $200 \mathrm{bp}$ of the interstitial tract in the rad51- $\Delta$ strain (Fig. 5B), when compared to the wild type distribution shown in Fig. 3C. This skew in the pattern of Ade ${ }^{-}$sectors suggests that association of telomerase with a fork that was formed in the absence of Rad51 resulted in a toxic DNA intermediate that could not be resolved prior to mitosis (see SI Appendix, Fig. S6). In contrast, telomerase-mediated elongation of the newly exposed interstitial tract in subsequent cell divisions (red arrows, Fig. $5 C$ ) was unaffected in the rad51- $\Delta$ strain. This argues that the ability of telomerase to recognize a newly collapsed fork vs. a fully replicated chromosome terminus was differentially regulated by Rad51.

The reduced ability of telomerase to elongate a newly collapsed fork at the interstitial tract also impacted native telomere length, as shown by a rad51- $\Delta$ telomere length profile for the Chr VI-R telomere (Fig. 5D and SI Appendix, Table S5). In the rad51- $\Delta$ strain, the sub-population of shorter-than-average telomeres were markedly reduced in length, when compared to the wild type Chr VI-R profile (superimposed in black on the rad51- $\Delta$ profile in Fig. $5 D$ ); telomeres that were less than $100 \mathrm{bp}$ in length rose to $22.3 \%$, compared to $9.9 \%$ in wild type $(p=0.0002)$. In contrast, the sub-population of full-length Chr VI-R telomeres were unaffected by the loss of Rad51 function. In addition, the number of rad51- $\Delta$ telomeres that failed to be elongated by telomerase increased from $11.5 \%$ in wild type to $20.9 \%$ in rad51- $\Delta$ (Fig. $5 E$ ). We propose that this disruption of telomere homeostasis is a direct consequence of the reduced ability of telomerase to successfully elongate collapsed replication forks at native telomeres in the rad51- $\Delta$ strain, as anticipated by the Rad51-dependent behavior at the interstitial tract (Fig. 5B and 5C). A role for Rad51 in regulating telomerase, which was not predicted by previous models, provides further support for the contribution of replication fork collapse to wild type telomere length homeostasis. Moreover, this indicates that telomerase activity at newly collapsed forks proceeds 
through a regulatory pathway that is distinct from how telomerase engages fully replicated chromosome termini. Specifically, the above observations suggest that the structure of the collapsed fork, dictated in part by remodeling proteins like Rad51, plays a role in regulating telomerase activity.

\section{Discussion}

A widely accepted assumption in telomere biology is that the primary substrate for telomerase in wild type cells is the G-rich overhang present at chromosome ends, produced following completion of DNA replication. In this study, we show that in wild type yeast, there is second substrate for telomerase generated by spontaneous replication fork collapse. We also report that the extensive addition of telomeric repeats at newly collapsed forks is notably different from the more limited telomerase activity observed at fully replicated yeast chromosome ends.

The observation that there are two substrates for telomerase suggests a re-interpretation of the long-standing model that telomerase preferentially elongates short telomeres (26-30). This model received substantial support from an examination of telomerase-mediated elongation of individual yeast chromosome termini during a single cell cycle, which revealed a remarkable length preference. Very few telomeres that retained $\geq 300$ bp of $\mathrm{G}_{1-3} \mathrm{~T}$ DNA were substrates for telomerase, whereas $\sim 50 \%$ of telomeres that were $\leq 100$ bp were elongated (27). This subpopulation of very short telomeres also underwent extensive elongation by telomerase (postulated to be due to a "loss of normal telomerase control"), in contrast to the much more limited synthesis at telomeres that were closer to physiological lengths. To explain these results, telomeres were proposed to switch between telomerase-extendible and telomerase-nonextendible states, with the "switch" determined by the length of the duplex telomeric tract (27).

We propose that these two states are instead structurally and temporally distinct substrates for telomerase, generated by two different processes (fork collapse vs. completion of DNA replication). Thus, we suggest that the preference observed in prior studies (27) was not the 
result of a feedback mechanism that directed telomerase to a subset of fully replicated termini, but rather a choice by telomerase between two different categories of substrates. The prior model was also based on the assumption that wild type telomeres are maintained around a fixed average length (30); however, this is contradicted by our high resolution analysis showing that the steady state lengths of individual chromosome ends in wild type cells span as much as $450 \mathrm{bp}$, with $\sim 25 \%$ of termini diverging from the mean (either shorter or longer) by more than $100 \mathrm{bp}$.

A new model for telomere length regulation. We propose a new model in which the activity of telomerase in response to spontaneous fork collapse is a major determinant of telomere length regulaton. This model does not invoke a length-sensing mechanism. Instead, it stems from the premise that wild type telomere homeostasis is defined by both average telomere length as well as the ability to tolerate substantial length variation. We propose that these two parameters are maintained by a dynamic balance between two opposing events: DNA replication failure vs. telomerase elongation of the resulting fork collapse. Length variation arises due to the combined impact of fork collapse events that escape elongation by telomerase (resulting in potentially significant sequence loss in just one cell division) as well as collapsed forks that are elongated (often extensively in a single cell division). This predicts that the extent of length variation would be altered by either increasing the number of fork collapse events, as we show for the cdc13F539A strain, or decreasing the ability of telomerase to elongate newly collapsed forks, as we also show for rad51- $\Delta$.

Mechanistically, we propose that these two opposing forces - sequence loss due to fork collapse vs. sequence addition due to telomerase - are linked by the t-RPA complex, which associates with the replication fork to perform two activities. The t-RPA contributes to progression of the replisome through duplex telomeric DNA, thereby minimizing sequence loss, but it also recruits telomerase to the fork, thereby increasing the probability of extensive re-elongation when DNA replication fails. These two activities are supported by our demonstration that Cdc13 associates with duplex telomeric DNA even in the absence of a single-stranded terminus, and that elongation 
of newly collapsed forks is dependent on the Cdc13 telomerase recruitment activity.

This proposal also extends an elegant model first put forward by Greider (31), who proposed that telomerase is delivered to chromosome ends by traveling with the replication fork. She suggested that the probability of telomerase dissociation from the fork increased with the length of the duplex telomeric tract, thereby providing a mechanism for depositing telomerase more frequently at shorter telomeres. Although this model assumed that there was only a single substrate for telomerase, this concept could easily be expanded so that association with the replication fork would provide a single mechanism for delivering telomerase to both categories of substrates. Greider's proposal that telomerase association with the fork decreases during fork progression is supported by our observation that elongation of newly collapsed forks at the interstitial telomeric tracts showed a gradient, with proximal fork collapse events more likely to be elongated by telomerase than more distal collapsed forks.

An obvious question is how frequently replication fork collapse occurs at native chromosome ends. Fork collapse at the interstitial tract occurs only every $\sim 1200$ cell divisions, which if extrapolated to chromosome ends, would be too low to have an impact on any aspect of telomere homeostasis. However, several genetic observations suggest that replication fork collapse impacts chromosomes ends: in mutant strains that either reduced the ability of telomerase to elongate a newly collapsed fork (rad51- $\Delta$ ) or increased in the frequency of fork collapse (cdc13F538A) at the interstitial tract, telomere homeostasis at chromosome ends was also altered, indicating that fork collapse occurs at a substantially higher frequency at the ends of chromosomes than at the interstitial tract. We speculate that an deceased ability of fork stabilization systems to prevent fork collapse and/or re-load replisomes onto stalled or collapsed forks during the very last stages of replicating the genome may lead to an increase in DNA replication errors, relative to the behavior at high efficiency ARS elements such as ARS910 (46), immediately adjacent to the interstitial tract on Chr IX. 


\section{What is the structure of the newly collapsed fork that is recognized by telomerase? Once} a fork stalls during replication of duplex telomeric DNA, there are several options for how the fork could be re-modeled to produce a 3' single-stranded G-rich substrate for telomerase (illustrated in SI Appendix, Fig. S6). If the stalled fork is converted into a double-strand break (DSB), subsequent elongation of the two DNA ends by telomerase will yield non-identical chromosomes in the $\mathrm{Ade}^{-}$founder cell, which can be easily distinguised from each other due to the irregular repeat synthesized by yeast telomerase. Notably, the pattern predicted for a DSB (a 50:50 inheritance pattern by progeny due to two founder chromosomes; SI Appendix, Fig. S6) was observed less than $1 \%$ of the time among the $>300 \mathrm{Ade}^{-}$sectors analyzed during this study. Instead, our data showed that more that $50 \%$ of $\mathrm{Ade}^{-}$half-sectors were founded by a single $\mathrm{Ade}^{-}$ founder chromosome, indicative of a single DNA end that was elongated by telomerase. This invokes two options by which a stalled replication fork could give rise to a single DNA end. A DNA nick in one strand of the stalled fork (either present in duplex telomeric DNA prior to replication or introduced after fork stalling) could create a so-called "one-sided DSB" that could be elongated by telomerase (SI Appendix, Fig. S6). A second option is fork regression, as illustrated in Fig. 5A. Although fork reversal was initially viewed solely as a pathological response in checkpoint-deficient strains (64), more recent findings have suggested that fork regression is utilized as a response to replication stress, by pausing forks to prevent genomic instability (65). At telomeres, regressed forks may perform an additional specialized function as a substrate for telomerase. In yeast, the evidence for a regressed fork as a potential telomerase substrate has largely relied on methods such as 2-D gels that detect the accumulation of replication intermediates in a population of cells, which is usually performed under conditions of replication stress $(39,40)$; however, this does not differentiate between whether telomerase acted in the same cell division or in subsequent cell divisions, after the regressed fork has been resolved. In contrast, the assay we used here provides a molecular snapshot of telomerase activity in the same cell division as fork collapse occurs, and also allows an examination of events in unstressed cells. 
A role for telomerase at replication forks also argues that previously identified regulators of telomere length may exert their effects at collapsed forks, rather than fully replicated termini. For example, the role of the Mre11-Rad50-Xrs2 (MRX) complex in yeast telomere length regulation has long been assumed to be due to an end-processing activity at fully replicated termini $(66,67)$; however, increasing evidence for a function for this complex at stalled replication forks suggests that MRX may regulate telomere length homeostasis by protecting fork integrity (68-70). This also argues for an alternative interpretation of the recent observation that there is a pathological interaction between replication forks and telomerase in RTEL1 -/- mammalian cells (71). We suggest that collapsed forks generated in the absence of the RTEL1 helicase may be structurally incompatible as a telomerase substrate, resulting in an unproductive interaction between telomerase and collapsed forks that is specific to the RTEL1 defect. Such a model would explain the impaired elongation of the shortest sub-population of chromosome ends in RTEL1 -/- cells (72), due to a presumed inability of telomerase to recognize and elongate these atypical collapsed forks.

A challenge to the end protection model. This study also re-assessed a long-standing model for the role of the t-RPA complex at yeast telomeres, which initiated with a pivotal observation showing that Cdc13-depleted cells accumulated single-stranded regions in telomere-proximal regions of the genome (73). To explain these DNA lesions, Hartwell and colleagues put forth two alternative models: Cdc13 protected chromosome termini from resection by 5'-to-3' exonucleases (the "capping" or end protection model), or Cdc13 instead was required for lagging strand replication of telomeric DNA. A series of papers drove the first model, supported in part by in vivo suppression of the $c d c 13-1$ defect by an exo1- $\Delta$ mutation which was assumed to be due to Exo1mediated degradation of chromosome ends (74-77). Although the end protection model for the budding yeast Cdc13-Stn1-Ten1 complex has largely dominated for the past 20 years, an increasing body of evidence in numerous other species indicates that this conserved RPA-like complex promotes lagging strand DNA replication through its association with the Pol $\alpha /$ primase 
complex $(21-23,51,52)$. In addition, more recent studies have questioned whether the increased levels of ssDNA in a $c d c 13-1$ strain, as well as $\operatorname{stn} 1^{-}$and $\operatorname{ten} 1^{-}$strains, were terminus-specific, as this DNA lesion was not sensitive to pre-treatment of genomic DNA with ssDNA-specific exonucleases that depend on an exposed 3 ' terminus $(78,79)$.

This suggests an alternative explanation for the enhanced levels of ssDNA observed in a cdc13-1 strain, which is that stalled replication forks due to impaired Cdc13 function lead to an Exo1dependent accumulation of ssDNA on the lagging strand. Consistent with a role in duplex DNA replication, we show that fork collapse is substantially elevated in cdc13-impaired strains; additionally, this high rate of fork collapse is partly suppressed when EXO1 is deleted, consistent with the previously observed stabilization of stalled replication forks in the absence of Exol (5356). We therefore suggest that the essential function of the t-RPA complex is to promote replication of duplex telomeric DNA, rather than to protect termini from resection.

\section{Materials and Methods}

Yeast genetic methods. Standard methods were used for yeast strain construction and propagation; all strains used in this study (SI Appendix Table S6) were isogenic. The yeast strain used to monitor replication fork collapse at an interstitial telomeric tract was constructed by inserting a terminal fragment from the Chr I-L telomere (composed of $302 \mathrm{bp}$ of sub-telomeric DNA and 390 bp of $\mathrm{G}_{1-3}$ T DNA) at a site on Chr IX that was $6.7 \mathrm{~kb}$ distal to ARS922 and $27 \mathrm{~kb}$ upstream of the Chr IX terminus, as a linearized plasmid (pVL7292) containing the $692 \mathrm{bp} \mathrm{Chr} \mathrm{I-L}$ telomere and selectable markers, flanked by homology to the target location. The ADE2 gene was introduced on the distal portion of Chr IX (14.4 kb from the terminus), accompanied by a corresponding deletion of the endogenous $A D E 2$ locus. The integrity of the interstitial $\mathrm{G}_{1-3} \mathrm{~T}$ telomeric DNA tract was confirmed after each strain construction by PCR amplification and sequence analysis. Replication fork collapse was monitored by plating early log phase cells (O.D. 0.3) for single colonies at low density ( $\leq 150$ colonies/plate) on rich media that was limiting for adenine. Plates were incubated at $30^{\circ}$ until colonies were fully grown, plus an additional $\sim 5$ 
days at room temperature to allow $\mathrm{Ade}^{-}$sectors to become readily detectable. The frequency of fork collapse that occurred during the first cell division was determined by the number of $\mathrm{Ade}^{-}$half-sectors, relative to the total number of colonies. This approach directly measures the frequency of events per single cell division, in contrast to assays that measure the accumulation of events in a population of cells in a liquid culture (which employ statistical tests such as fluctuation analysis to calculate the probability of a mutation occurring per cell division). Fischer's exact test was used to determine two-sided $p$-values for the replication fork collapse assays. In experiments that monitored the introduction of mutant telomeric repeats, a mutant telomerase RNA under control of the GAL promoter (with the wild type template 3'-CACACCCACACCAC-5' changed to 3'-CACACCucCACCAC-5'; pVL6294) was transiently expressed in liquid culture containing galactose for four hours, followed by plating on rich media containing glucose (which shuts down the GAL promoter in subsequent cell divisions).

PCR amplification, cloning and sequencing of telomeres. Yeast genomic DNA was prepared from $1 \mathrm{ml}$ cultures grown in rich media from single colonies or $\mathrm{Ade}^{-}$half-sectors using the Wizard $®$ Genomic DNA Purification Kit (Promega). Genomic DNA (150 ng) was treated with terminal transferase in the presence of $1 \mathrm{mM} \mathrm{dCTP}$ to introduce a poly $(C)_{n}$ tract at chromosome ends, as previously described (80). Chr I-L and Chr VI-R were amplified using primers that recognized the sub-telomeric region of Chr I-L (5'-GCGGTACCAGGGTTAGATTAGGGCTG-3') or Chr VI-R (5'-ACGTGTGCGTACGCCATATCAATATGC-3') and the poly $(C)_{\mathrm{n}}$ tract (5'CGGGATCCGGGGGGGGGGGGGGGGGG-3'). Genomic DNA + primers were denatured at $94^{\circ} \mathrm{C}$ and cycled 35 times, with annealing $\left(20 \mathrm{sec}, 60^{\circ} \mathrm{C}\right)$ and extension $\left(90 \mathrm{sec}, 72^{\circ} \mathrm{C}\right)$ for each cycle, followed by a final extension step $\left(10 \mathrm{~min}, 72^{\circ} \mathrm{C}\right)$. Two methods were used to prepare PCR products for cloning: (i) for telomere length profiles, PCR products were precipitated to ensure no bias with regard to the spectrum of sizes recovered; (ii) for analysis of $\mathrm{Ade}^{-}$sectors (where subpopulations of shorter-than-average telomeres were less informative), PCR products were gelpurified. PCR reactions recovered by either method were $T A$-cloned into the $\mathrm{pCR}^{\mathrm{TM}} 2.1$ vector, transformed into DH5 $\alpha$ E. coli and plated on pre-warmed LB-Carb plates containing X-gal (5- 
bromo-4-chloro-3-indolyl- $\beta$-D-galactopyranoside) and carbenicillin $(60 \mu \mathrm{g} / \mathrm{ml})$. Transformants were incubated for $16 \mathrm{hrs}$ at $36^{\circ} \mathrm{C}$, immediately inoculated into $2 \mathrm{ml}$ of LB-Carb pre-warmed to $36^{\circ}$ and grown to saturation at $36^{\circ}$. All steps involving passage through E. coli were monitored to avoid temperature fluctuations, as empirical observations suggested that temperature shifts during E. coli propagation increased the frequency of deletions in the telomeric insert. Plasmid DNA was prepared using the QIAprep Spin Miniprep Kit (Qiagen), and the $\mathrm{G}_{1-3} \mathrm{~T}$ insert was sequenced by Eton Bioscience, Inc. using a protocol optimized for difficult-to-sequence templates. Cloned Chr I-L and Chr VI-R telomeres were sequenced using M13 Forward (-40) primer, which exploited the strong cloning bias of the $\mathrm{G}_{1-3} \mathrm{~T}$ insert with regard to orientation ( $\geq$ $95 \%$ of inserts were recovered in the same orientation). Telomeres generated following fork collapse at the interstitial telomeric tract on Chr IX were sequenced with a primer that recognized a unique sequence upstream of the interstitial tract (5'-TTCCCAGTCACGACGTTGTAAAACG-3'). The fidelity of the single-nucleotide sequence analysis of cloned telomeres was evaluated by two reconstruction experiments. Sequencing fidelity was assessed by duplicate sequence analysis (as independent sequence runs) of 16 clones with $\mathrm{G}_{1-3} T$ insert sizes ranging in size from 249 to $536 \mathrm{bp}$, for a total of 5,475 bp (SI Appendix Fig. S2). Re-sequencing detected 4 errors, due to mis-reads of GGG as GGGG at the end of the read for all 4 errors, resulting in a missense sequencing error rate of $0.07 \%$; no deletions due to sequence mis-reads were identified. The error rate during PCR amplification and/or propagation through E. coli was evaluated by PCR amplification and sequencing of the interstitial tract (240 bp of sub-telomeric DNA and $390 \mathrm{bp}$ of $\mathrm{G}_{1-3} \mathrm{~T} D N A$, from the yeast strain $\mathrm{YVL} 4836$ ), employing the same protocol used to analyze native telomeres. A total of 170 sequenced clones from 24 independent amplifications (3 PCR reactions from each of 8 yeast colonies) were analyzed, corresponding to 40,800 bp and 66,300 bp of subtelomeric and telomere DNA, respectively; error frequencies for different categories of mutations are summarized in SI Appendix Fig. S2. The highest error rate was due to single base-pair mismatches $(0.2 \%$ and $0.3 \%$ for sub-telomeric and telomeric DNA, respectively); the frequency of deletions or duplications in telomeric sequences was $0.08 \%$, with the most common category 
$(0.03 \%)$ due to $2 \mathrm{bp}$ deletions in $(\mathrm{TG})_{\mathrm{n}}$ tracts. We note that our estimated error rate was 10 -fold lower than the error rate evaluated by a similar protocol in a prior study (35).

Analysis of sequence data. For each telomere length profile, isolates were recovered from genomic DNA prepared from multiple sibling single colonies, with three independent PCR reactions per genomic preparation to prevent an outlier from dominating a profile. Duplicate isolates were rare $(<1 \%)$, and one was removed if recovered from the same PCR reaction. Cloned telomeres that lacked the poly $(C)_{n}$ tract or contained a deletion in the junction between sub-telomeric and telomeric DNA were infrequent and eliminated, on the assumption that these were indicative of errors generated during PCR amplification or propagation through E. coli that compromised the integrity of the $\mathrm{G}_{1-3} \mathrm{~T}$ tract. Telomere sequences were aligned using MacVector, anchored by alignments of the sub-telomeric and vector sequences. Identification of common stretches of $G_{1-3} T D N A$ inherited by progeny telomeres relied on a relatively high gap penalty; specifically, gaps in the $\mathrm{G}_{1-3} \mathrm{~T}$ tract were introduced only if the homology downstream of the gap extended for $\geq 25 \mathrm{bp}$, thereby preventing the introduction of multiple gaps within a single telomere sequence that artifactually maximized the extent of shared sequences. This conservative gap penalty may slightly under-estimate the extent of identical sequences inherited by progeny. No limitations were placed on the length of the gap itself (as long as it did not encompass the subtelomeric/telomeric boundary), on the assumption that the gap occurred during duplex telomeric DNA replication in yeast; this was supported in part by the identification of multiple occurrences of deletions in sibling yeast colonies. Gaps were removed prior to constructing telomere length alignments (see example in SI Appendix Fig. S1), which was similarly based on the assumption that they were generated in yeast (and hence their removal would accurately report on the in vivo length of each individual yeast telomere) rather than during PCR amplification or propagation in E. coli; if this assumption is incorrect, this under-estimates telomere length for each telomere length profile by $\leq 1 \%$. These alignments were used to determine minimal length of the Chr I-L and Chr VI-R telomeric DNA in the cell that founded each colony (the "founder chromosome"), which was defined as the longest stretch of $\mathrm{G}_{1-3} \mathrm{~T}$ sequence shared by three sequenced 
telomeres from each colony; see SI Appendix, Fig. S1 and Tables S1 to S5. The average rate of loss of founder sequence DNA was calculated as (the length of founder $\mathrm{G}_{1-3} \mathrm{~T}$ DNA) - (average founder $\mathrm{G}_{1-3} \mathrm{~T}$ DNA length) $/ 28$ cell divisions = \# of bp lost per cell division. Kolmgorov Smirnov tests were used to compare telomere length profiles from different yeast strains.

\section{Acknowledgments}

We thank Ted Weinert and members of the Lundblad lab for experimental suggestions. This work was supported by National Institutes of Health grants T32 GM007240 (to C.M.R. and A.E.G.), R01 GM106060 and R01 AG011728 (to V.L.), R01 GM059414 (to D.S.W.) and P30 CA014195 (to the Salk Institute Cancer Center), NSF grants IIS-1254123, IIS-1724421 and IOS1556388 (to T.O.S), a Graduate Fellowship from the Glenn Center for Aging Research at the Salk Institute (to C.M.R.), the Joe W. and Dorothy Dorsett Brown Foundation (to V.L. and T.O.S) and the Becky and Ralph S. O'Connor Chair (to V.L.).

\section{References}

1. Palm W, de Lange T (2008) How shelterin protects mammalian telomeres. Annu. Rev. Genet. 42, 301-334.

2. Wu RA, Upton HE, Vogan JM, Collins, K (2017) Telomerase mechanism of telomere synthesis. Ann Rev Biochem 86, 439-460.

3. Caitlin MR, Artandi SE (2020) Regulation of human telomerase in homeostasis and disease. Nature Reviews Molec Cell Biol 21, 384-397.

4. Greider CW, Blackburn EH (1989) A telomeric sequence in the RNA of Tetrahymena telomerase required for telomere repeat synthesis. Nature 337, 331-337.

5. Wang F, Podell ER, Zaug AJ, Yang Y, Baciu P, Cech TR, Lei M (2007) The POT1-TPP1 telomere complex is a telomerase processivity factor. Nature 445, 506-510.

6. Zaug AJ, Podell ER, Nandakumar J, Cech TR (2010) Functional interaction between telomere protein TPP1 and telomerase. Genes Dev 24, 613-622.

7. Rao T, Lubin J, Armstrong GS, Tucey TM, Lundblad V, Wuttke DS (2014) Structure of Est3 reveals a bimodal surface with differential roles in telomere replication. Proc Natl Acad Sci USA 111, 214-218.

8. Friedman KL, Heit JJ, Long DM, Cech TR (2003) N-terminal domain of yeast telomerase reverse transcriptase: recruitment of Est3p to the telomerase complex. Mol Biol Cell 14, $1-13$.

9. Lee J, Mandell EK, Rao T, Wuttke DS, Lundblad V (2008) Investigating the role of the Est3 protein in yeast telomere replication. Nucleic Acids Res 38, 2279-2290.

10. Hsu M, Yu EY, Singh SM, Lue NF (2007) Mutual dependence of Candida albicans Est1p and Est3p in telomerase assembly and activation. Eukaryot Cell 6, 1330-1338.

11. Nandakumar J, Bell CF, Weidenfeld I, Zaug AJ, Leinwand LA, Cech TR (2012) The TEL patch of telomere protein TPP1 mediates telomerase recruitment and processivity. Nature 492, 285-289. 
12. Zhong FL, Batista LF, Freund A, Pech MF, Venteicher AS, Artandi SE (2012) TPP1 OBfold domain controls telomere maintenance by recruiting telomerase to chromosome ends. Cell 150, 481-494.

13. Bianchi A, Negrini S, Shore D (2004) Delivery of yeast telomerase to a DNA break depends on the recruitment functions of Cdc13 and Est1. Mol Cell 16, 139-146.

14. Gallardo F, Laterreur N, Cusanelli E, Ouenzar F, Querido E, Wellinger RJ, Chartrand P (2011) Live cell imaging of telomerase RNA dynamics reveals cell cycle-dependent clustering of telomerase at elongating telomeres. Mol Cell 44, 819-827.

15. Tucey TM, Lundblad V (2013) A yeast telomerase complex containing the Est1 recruitment protein is assembled early in the cell cycle. Biochemistry 52, 1131-1133.

16. Nugent C, Hughes TR, Lue NF, Lundblad V (1996) Cdc13p: A single-strand telomeric DNA-binding protein with a dual role in yeast telomere maintenance. Science 274,249 252

17. Gao H, Cervantes RB, Mandell EK, Otero JH, Lundblad V (2007) RPA-like proteins mediate yeast telomere function. Nat Struct Mol Biol 14, 208-214.

18. Gelinas AD, Paschini M, Reyes FE, Héroux A, Batey RT, Lundblad V, Wuttke DS (2009) Telomere capping proteins are structurally related to RPA with an additional telomerespecific domain. Proc Natl Acad Sci USA 106, 19298-19303.

19. Glustrom LW, Lyon KR, Paschini M, Reyes CM, Parsonnet NV, Toro TB, Lundblad V, Wuttke DS (2018) Single-stranded telomere-binding protein employs a dual rheostat for binding affinity and specificity that drives function. Proc Natl Acad Sci USA 115, 1031510320.

20. Mandell EK, Gelinas AD, Wuttke DS, Lundblad V (2011) Sequence-specific binding to telomeric DNA is not a conserved property of the Cdc13 DNA binding domain. Biochemistry 50, 6289-6291.

21. Goulian M, Heard CJ, Grimm SL (1990) Purification and properties of an accessory protein for DNA polymerase $\alpha /$ primase. J Biol Chem 265, 13221-13230.

22. Casteel DE, Zhuang S, Zeng Y, Perrino FW, Boss GR, Goulian M, Pilz RB (2009) A DNA polymerase $\alpha$ /primase cofactor with homology to replication protein A-32 regulates DNA replication in mammalian cells. J Biol Chem 284, 5807-5818.

23. Derboven E, Ekker H, Kusenda B, Bulankova P, Riha K (2014) Role of STN1 and DNA polymerase $\alpha$ in telomere stability and genome-wide replication in Arabidopsis. PLoS Genet 10, e1004682.

24. Nakaoka H, Nishiyama A, Saito M, Ishikawa F (2012) Xenopus laevis Ctc1-Stn1-Ten1 (xCST) protein complex is involved in priming DNA synthesis on single-stranded DNA template in Xenopus egg extract. J Biol Chem 287, 619-627.

25. Hom RA, Wuttke DS (2017) Human CST prefers G-Rich but not necessarily telomeric sequences. Biochemistry 56, 4210-4218.

26. Smogorzewska A, van Steensel B, Bianchi A, Oelmann S, Schaefer MR, Schnapp G, de Lange T (2000) Control of human telomere length by TRF1 and TRF2. Mol Cell Biol 20, 1659-1668.

27. Teixeira MT, Arneric M, Sperisen P, Lingner J (2004) Telomere length homeostasis is achieved via a switch between telomerase-extendible and -nonextendible states. Cell 117, 323-35.

28. Britt-Compton B, Capper R, Rowson J, Baird DM (2009) Short telomeres are preferentially elongated by telomerase in human cells. FEBS Lett $583,3076-3080$.

29. Marcand S, Gilson E, Shore D (1997) A protein-counting mechanism for telomere length regulation in yeast. Science $275,986-990$.

30. Bianchi A, Shore D (2009) Telomere length regulation: coupling DNA end processing to feedback regulation of telomerase. EMBO J 28, 2309-2322.

31. Greider CW (2016) Regulating telomere length from the inside out: the replication fork model. Genes Dev 30, 1483-1491.

32. Shampay J, Blackburn EH (1988) Generation of telomere-length heterogeneity in Saccharomyces cerevisiae. Proc Natl Acad Sci USA 85, 534-538. 
33. Baird DM, Britt-Compton B, Rowson J, Amso NN, Gregory L, Kipling D (2006) Telomere instability in the male germline. Hum Mol Genet 15, 45-51.

34. Chang M, Arneric M, Lingner J (2007) Telomerase repeat addition processivity is increased at critically short telomeres in a Tel1-dependent manner in Saccharomyces cerevisiae. Genes Dev 21, 2485-2494.

35. Claussin C, Chang M (2016) Multiple Rad52-mediated homology-directed repair mechanisms are required to prevent telomere attrition-induced senescence in Saccharomyces cerevisiae. PLOS Genetics 12, e1006176.

36. Dionne I, Wellinger RJ (1998) Processing of telomeric DNA ends requires the passage of a replication fork. Nucleic Acids Res 26, 5365-5371.

37. Chai W, Du Q, Shay JW, Wright WE (2006) Human telomeres have different overhang sizes at leading versus lagging strands. Mol Cell 21, 427-435.

38. Miller KM, Rog O, Cooper JP (2006) Semi-conservative DNA replication through telomeres requires Taz1. Nature 440, 824-828.

39. Dehé PM, Rog O, Ferreira MG, Greenwood J, Cooper JP (2012) Taz1 enforces cell-cycle regulation of telomere synthesis. Mol Cell 46, 797-808.

40. Matmati S, Lambert S, Géli, V, Coudon S (2020) Telomerase repairs collapsed replication forks at telomeres. Cell Reports 30, 3312-3322.

41. Hardy J, Churikov D, Geli V, Simon M-N (2014) Sgs1 and Sae2 promote telomere replication by limiting accumulation of ssDNA. Nature Comm 5, 5004.

42. Singer MS, Gottschling DE (1994) TLC1: template RNA component of Saccharomyces cerevisiae telomerase. Science 266, 404-409.

43. Soudet J, Jolivet P, Teixeira MT (2014) Elucidation of the DNA end-replication problem in Saccharomyces cerevisiae. Mol Cell 53, 954-964.

44. Noël JF, Wellinger RJ (2011) Abrupt telomere losses and reduced end-resection can explain accelerated senescence of Smc5/6 mutants lacking telomerase. DNA Repair 10, 271-282.

45. Chang M, Luke B, Kraft C, Li Z, Peter M, Lingner J, Rothstein R (2009) Telomerase is essential to alleviate Pif1-induced replication stress at telomeres. Genetics 183, 779-791.

46. McGuffee SR, Smith DJ, Whitehouse I (2013) Quantitative, genome-wide analysis of eukaryotic replication initiation and termination. Mol Cell 50, 123-135.

47. Desany BA, Alcasabas AA, Bachant JB, Elledge SJ (1998) Recovery from DNA replicational stress is the essential function of the S-phase checkpoint pathway. Genes Dev 12, 2956-2970.

48. Lopez M, Cotta-Ramusino C, Pellicioli A, Liberi G, Plevani P, Muzi-Falconi M, Newlon CS, Foiani M (2001) The DNA replication checkpoint response stabilizes stalled replication forks. Nature 412, 557-561.

49. Sfeir A, Kosiyatrakul ST, Hockemeyer D, MacRae SL, Karlseder J, Schildkraut CL, de Lange T (2009) Mammalian telomeres resemble fragile sites and require TRF1 for efficient replication. Cell 138, 90-103.

50. Förstemann K, Lingner J (2001) Molecular basis for telomere repeat divergence in budding yeast. Mol Cell Biol 21, 7277-7286.

51. Takikawa M, Tarumoto $Y$, Ishikawa $F(2017)$ Fission yeast Stn1 is crucial for semiconservative replication at telomeres and subtelomeres. Nucleic Acids Res 45, 12551269.

52. Gu P, Min JN, Wang Y, Huang C, Peng T, Chai W, Chang S (2012) CTC1 deletion results in defective telomere replication, leading to catastrophic telomere loss and stem cell exhaustion. EMBO J 31, 2309-2321.

53. Cotta-Ramusino C, Fachinetti D, Lucca C, Doksani Y, Lopes M, Sogo J, Foiani M (2005) Exo1 processes stalled replication forks and counteracts fork reversal in checkpointdefective cells. Mol Cell 17, 153-159.

54. Tsang E, Miyabe I, Iraqui I, Zheng J, Lambert SAE, Carr AM (2014) The extent of errorprone replication restart by homologous recombination is controlled by Exo1 and checkpoint proteins. J Cell Sci 127, 2983-2994. 
55. Larsen NB, Liberti SE, Vogel I, Jørgensen SW, Hickson ID, Mankouri HW (2017) Stalled replication forks generate a distinct mutational signature in yeast. Proc Natl Acad Sci USA 114, 9665-9670.

56. Segurado M, Diffley JF (2008) Separate roles for the DNA damage checkpoint protein kinases in stabilizing DNA replication forks. Gene Dev 22, 1816-1827.

57. Paschini M, Toro TB, Lubin JW, Braunstein-Ballew B, Morris DK, Lundblad V (2012) A naturally thermolabile activity compromises genetic analysis of telomere function in Saccharomyces cerevisiae. Genetics 191, 79-932.

58. Higgins NP, Kato K, Strauss B (1976) A model for replication repair in mammalian cells. J Mol Biol 101, 417-425.

59. Rickman K, Smorgorzewska A (2019) Advances in understanding DNA processing and protection at stalled replication forks. J Cell Biol 218, 1096-1107.

60. Bhat KP, Krishnamoorthy A, Dungrawala H, Garcin EB, Modesti M, Cortez D (2018) RADX Modulates RAD51 Activity to Control Replication Fork Protection. Cell Reports 24, 538-545.

61. Ait Saada A, Teixeira-Silva A, Iraqui I, Costes A, Hardy J, Paoletti G, Fréon K, Lambert SAE (2017) Unprotected replication forks are converted into mitotic sister chromatid bridges. Mol Cell 66, 398-410.

62. Hashimoto Y, Ray Chaudhuri A, Lopes M, Costanzo V (2010) Rad51 protects nascent DNA from Mre11-dependent degradation and promotes continuous DNA synthesis. Nat Struct Mol Biol 17, 1305-1311.

63. Zellweger R, Dalcher D, Mutreja K, Berti M, Schmid JA, Herrador R, Vindigni A, Lopes M (2015) Rad51-mediated replication fork reversal is a global response to genotoxic treatments in human cells. J Cell Biol 208, 563-579.

64. Sogo JM, Lopes M, Foiani M (2002) Fork reversal and ssDNA accumulation at stalled replication forks owing to checkpoint defects. Science 297, $599-602$.

65. Neelsen KJ, Lopes M (2015) Replication fork reversal in eukaryotes: from dead end to dynamic response. Nat Rev Mol Cell Biol 16: $207-220$

66. Larrivée M, LeBel C Wellinger RJ (2004) The generation of proper constitutive G-tails on yeast telomeres is dependent on the MRX complex. Genes Dev 18, 1391-1396.

67. Martina M, Clerici M, Baldo V, Bonetti, D, Lucchini G, Longhese MP (2012) A balance between Tel1 and Rif2 activities regulates nucleolytic processing and elongation at telomeres. Mol Cell Biol 32, 1604-1617.

68. Costanzo V (2011) Brca2, Rad51 and Mre11: performing balancing acts on replication forks. DNA Repair 10, 1060-1065.

69. Bentsen IB, Nielsen I, Lisby M, Nielsen HB, Gupta SS, Mundbjerg K, Andersen AH, Bjergbaek $L$ (2013) MRX protects fork integrity at protein-DNA barriers, and its absence causes checkpoint activation dependent on chromatin context. Nucleic Acids Res.41, 3173-3189.

70. Menin L, Ursich S, Trovesi C, Zellweger E, Lopes M, Longhese MP, Clerici M (2018) Tel1/ATM prevents degradation of replication forks that reverse after topoisomerase poisoning. EMBO reports 19, e45535.

71. Margalef P, Kotsantis P, Borel V, Bellelli R, Panier S, Boulton SJ (2018) Stabilization of reversed replication forks by telomerase drives telomere catastrophe. Cell 172, 439-453.

72. Ding H, Schertzer M, Wu X, Gertsenstein M, Selig S, Kammori M, Pourvali R, Poon S, Vulto I, Chavez E, Tam PP, Nagy A, Lansdorp PM (2004) Regulation of murine telomere length by Rtel: an essential gene encoding a helicase-like protein. Cell 117, 873-886.

73. Garvik B, Carson M, Hartwell L (1995) Single-stranded DNA arising at telomeres in cdc13 mutants may constitute a specific signal for the RAD9 checkpoint. Mol Cell Biol 15, 61286138.

74. Lydall D, Weinert T (1995) Yeast checkpoint genes in DNA damage processing: implications for repair and arrest. Science 270, 1488-1491. 
75. Booth C, Griffith E, Brady G, Lydall D (2001) Quantitative amplification of single-stranded DNA (QAOS) demonstrates that cdc13-1 mutants generate ssDNA in a telomere to centromere direction. Nucleic Acids Res 29, 4414-4422.

76. Maringele L, Lydall D (2002) EXO1-dependent single-stranded DNA at telomeres activates subsets of DNA damage and spindle checkpoint pathways in budding yeast yku70 $\Delta$ mutants. Genes Dev 16, 1919-1933.

77. Zubko MK, Guillard S, Lydall D (2004) Exo1 and Rad24 differentially regulate generation of ssDNA at telomeres of Saccharomyces cerevisiae cdc13-1 mutants. Genetics 168, 103-115.

78. Petreaca RC, Chiu HC, Nugent Cl (2007) The role of Stn1p in Saccharomyces cerevisiae telomere capping can be separated from its interaction With Cdc13p. Genetics 177, 1459-1474.

79. Xu L, Petreaca RC, Gasparyan HJ, Vu S, Nugent CI (2009) TEN1 is essential for CDC13-mediated telomere capping. Genetics 183, 793-810.

80. Forstemann K, Lingner $\mathrm{J}$ (2001) Molecular basis for telomere repeat divergence in budding yeast. Mol. Cell. Biol. 21, 7277-7286. 

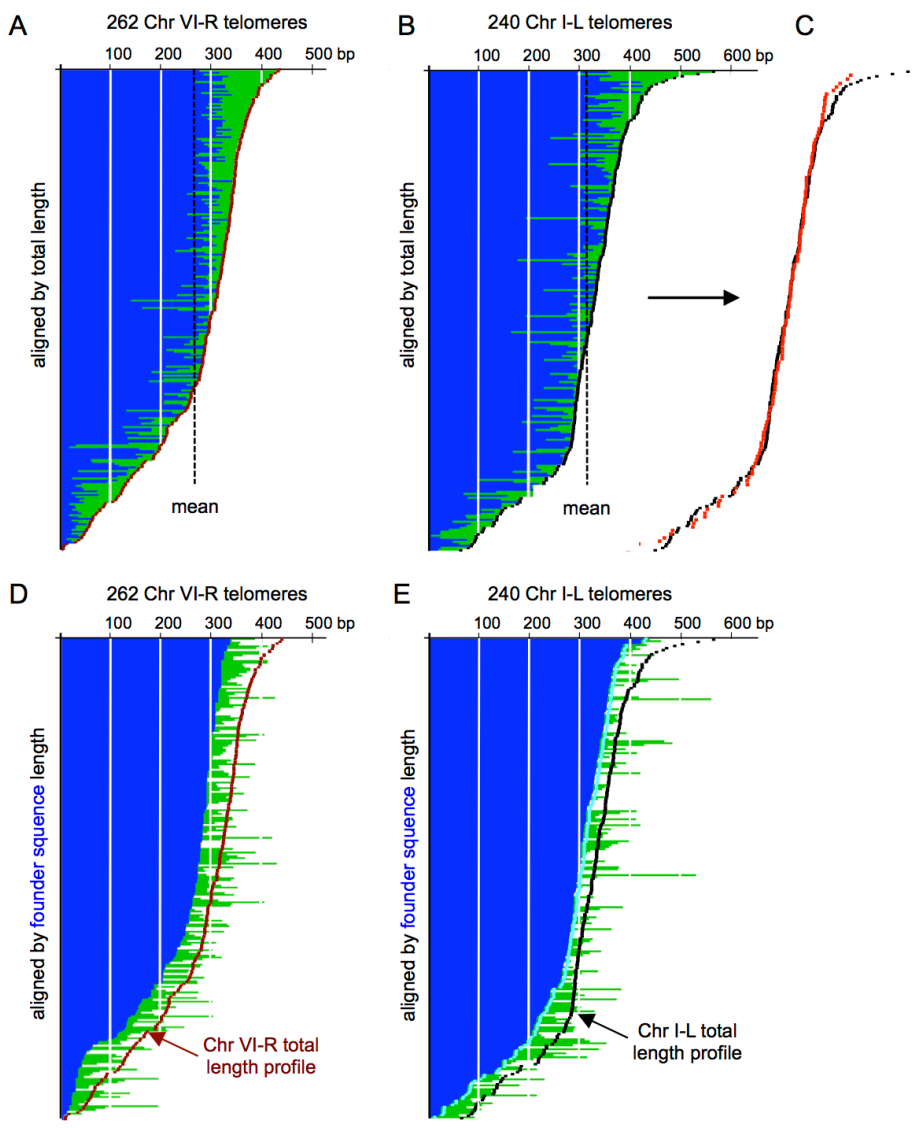

Figure 1. Wild type yeast telomeres exhibit extensive length variation. ( $A$ and $B$ ) Sequences of Chr VI-R and Chr I-L telomeres (262 or 240 isolates, respectively) from three single colonies aligned based on overall length; mean length was 270 and $311 \mathrm{bp}$, respectively. $\mathrm{G}_{1-3} \mathrm{~T}$ founder sequence DNA is indicated in blue; green corresponds to DNA synthesized by telomerase. (C) The Chr I-L telomere length profile from Fig. $1 B$ (in black), compared to an independent assessment from 135 sequenced Chr I-L telomeres (in red); these two profiles are not statistically different $(p=0.65)$, as determined by a Kolmogorov Smirnov test. ( $D$ and $E$ ) The Chr VI-R and Chr I-L telomeres shown in $(A)$ and $(B)$, realigned based on the length of founder sequence DNA, with representations of the length profiles from $(A)$ and $(B)$ in brown and black, respectively, superimposed on the profiles in $(D)$ and $(E)$. Primary sequence data for Chr I-L and Chr VI-R telomeres are in SI Appendix, Tables S1 and S2, respectively, and the protocol for constructing alignments of sequenced telomeres and analysis of the fidelity of single-nucleotide sequencing are described in Materials and Methods and SI Appendix, Fig. S1 and Fig. S2. 
A

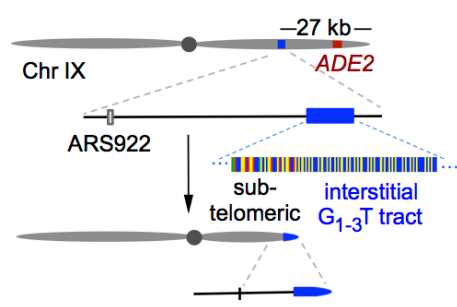

B

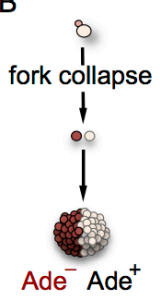

C

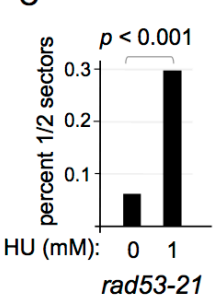

D

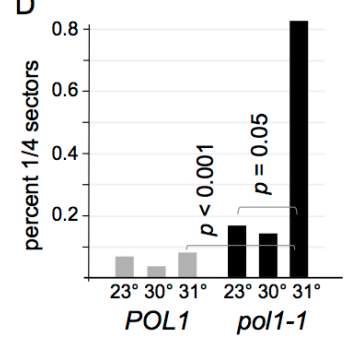

E

F sub-telomeric

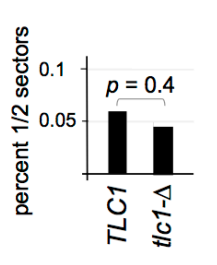
sequence

50
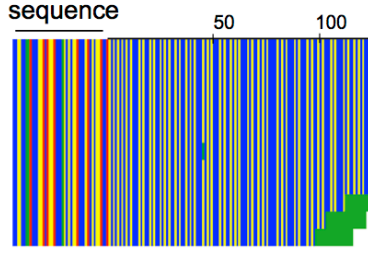

100

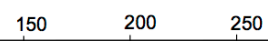

$300 \quad 350$ 350
Interstitial
$\mathrm{G}_{1-3} T$ tract

Progeny from an $\mathrm{Ade}^{-}$half-

sector from a t/c1- $\Delta$ strain

Figure 2. Spontaneous replication fork collapse at an interstitial telomeric tract. $(A)$ Structure of a modified version of Chr IX with an interstitial telomeric tract; see SI Appendix, Fig. S3 for more details. $(B)$ Illustration of an $A_{d e}{ }^{-}$half-sectored colony from the yeast strain with the modified Chr IX shown in $(A)$, due to a spontaneous replication fork collapse that occurred during the first cell division; the $\mathrm{Ade}^{-}$half of the colony can be detected visually due to accumulation of a red pigment from oxidation of an intermediate in the adenine biosynthetic pathway. For simplicity, fork collapse events in subsequent cell divisions that produce 1/4 sectors, 1/8 sectors, etc. are not indicated. $(C)$ Frequency of sectored colonies in a rad53-21 strain in the presence or absence of $1 \mathrm{mM} \mathrm{HU}$ (12,905 and 7,084 colonies, respectively). (D) Frequency of sectored colonies in POL 1 vs. pol1-1 strains at $23^{\circ}, 30^{\circ}$ or $31^{\circ} \mathrm{C}$, with $\sim 2,000$ to 2,500 colonies examined for each genotype at each temperature; since the effects of the pol1-1 mutation did not become pronounced until the second cell division at semi-permissive temperatures, the frequency of Ade ${ }^{-}$ quarter-sectors was evaluated in this experiment. $(E)$ Frequency of half-sectored colonies in wild type vs. t/c1- $\Delta$ strains (23,063 and 38,180 colonies, respectively). ( $F$ ) An alignment of sequenced telomeres from the progeny of an $A_{d e}{ }^{-}$half-sector from a t/c1- $\Delta$ strain, following replication fork collapse at the interstitial tract; $\mathrm{G}=$ blue, $\mathrm{T}=$ yellow, $\mathrm{C}=$ green, $\mathrm{A}=$ red. 


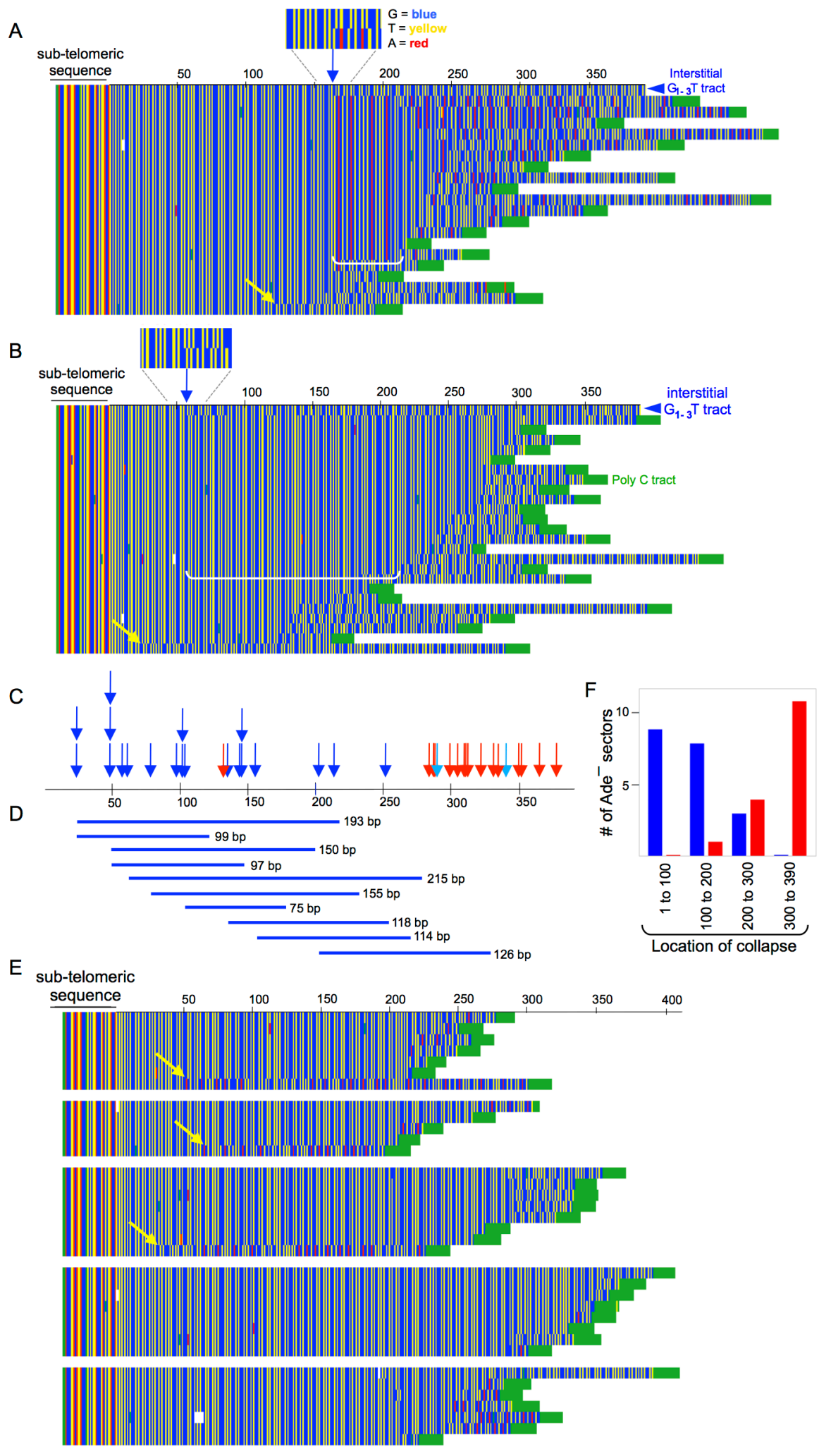

Figure 3. Telomerase elongates collapsed replication forks (legend next page). 
Figure 3. Telomerase elongates collapsed replication forks. $(A)$ and $(B)$ Alignment of sequenced telomeres from two Ade ${ }^{-}$half-sectors reveals the sites (indicated by blue arrows) at which telomerase initiated synthesis at nucleotides 163 and 57, respectively; the bracket defines the extent of elongation by telomerase in the first cell division. The half-sector in $(A)$ was identified following transient expression of a telomerase with a mutant telomerase RNA template (see Materials and Methods for details), whereas the half-sector in $(B)$ was recovered from a wild type strain. See $(E)$, below, for an explanation of the yellow arrows. (C) A map of the $390 \mathrm{bp}$ interstitial telomeric tract, showing the position at which telomerase initiated synthesis in $38 \mathrm{Ade}^{-}$ half-sectored colonies in a wild type strain; blue arrows correspond to sectors where telomerase elongated the newly exposed Chr IX terminus in the $\mathrm{Ade}^{-}$founder cell (i.e. the same cell division in which the fork collapse occurred), whereas red arrows correspond to sectors in which the Chr IX terminus was elongated in a subsequent cell division. Light blue arrows indicate sectors in which telomerase possibly elongated the collapsed fork in the first cell division, but this could not be determined unambiguously based on the pattern of inheritance by progeny; see example in $S I$ Appendix, Fig. S9. (D) The extent of telomerase synthesis in the $\mathrm{Ade}^{-}$founder cell division for 10 "blue arrow" Ade ${ }^{-}$half-sectors from the wild type strain; the extent of synthesis for all 20 "blue arrow" Ade ${ }^{-}$half-sectors is shown in SI Appendix, Fig. S8. (E) Alignments of the sequence of Chr I-L telomeres of progeny from five colonies from a wild type strain in which the modified telomerase was transiently expressed just prior to colony formation. Yellow arrows indicate the pattern predicted for a replication fork collapse followed by elongation by the modified telomerase; the yellow arrows in $(A)$ and $(B)$, above, are similarly consistent with a telomeraseelongated fork collapse. $(F)$ Graphical representation of the distribution across the $390 \mathrm{bp}$ interstitial tract of the two categories of sectors shown in $(C)$; the two ambiguous sectors indicated as light blue arrows in $(C)$ are not included. 

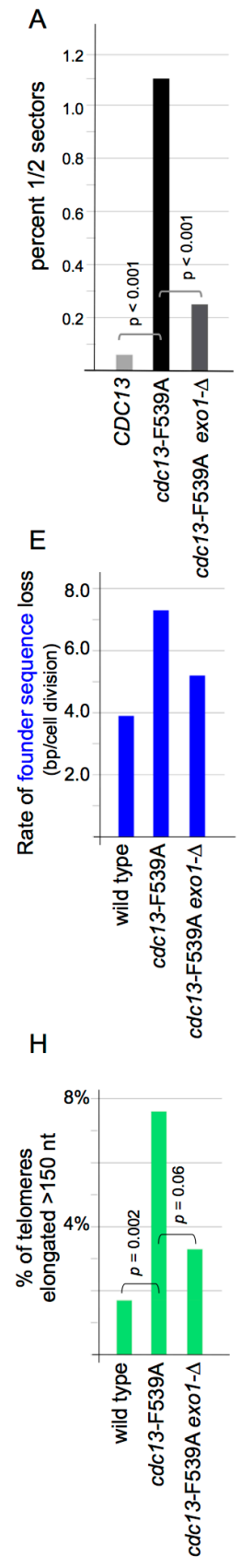

B sub-telomeric

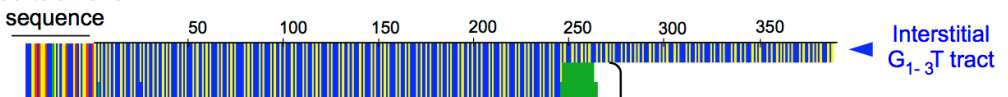

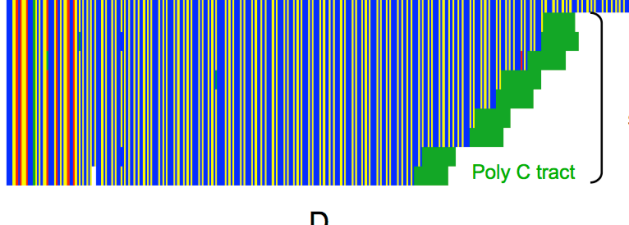

Progeny from an $\mathrm{Ade}^{-}$halfsector from an est1-60 strain

C

251 cdc13-F539A Chr I-L telomeres
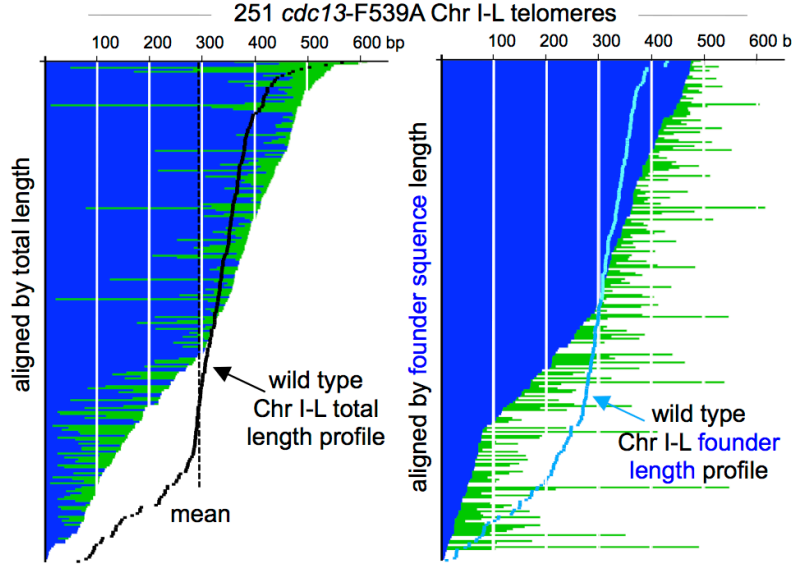

I 189 cdc13-F539A exo1- $\Delta$ Chr I-L telomeres

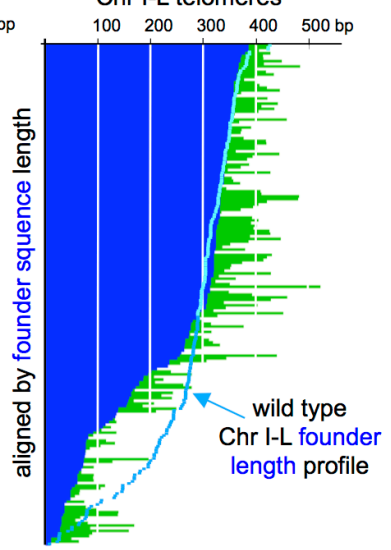

F 240 wild type Chr I-L telomeres $100 \quad 200300 \mathrm{bp}$

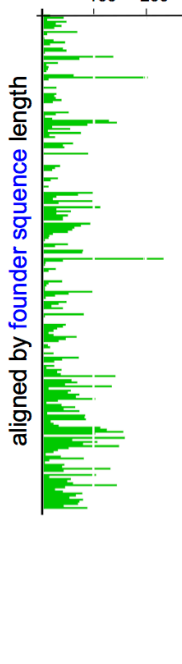

G
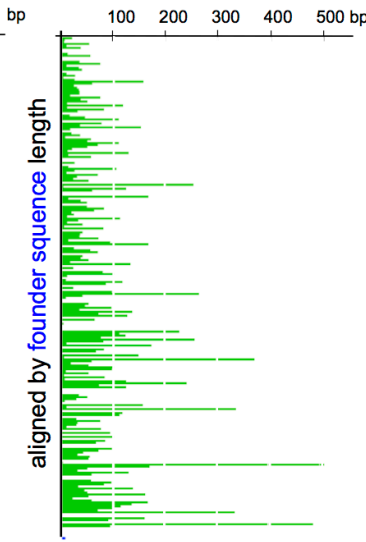

J 189 cdc13-F539A exo1- $\Delta \mathrm{K}$
Chr I-L telomeres
100200300 bp

wild type exo1- $\triangle$ cdc13-F539A exo1- $\Delta$ cdc13-F539A

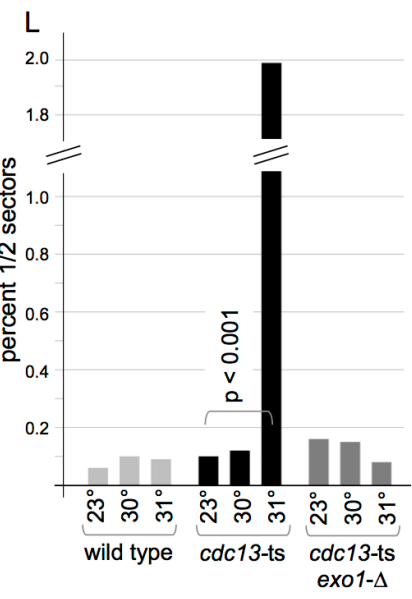

Figure 4. Replication fork collapse promotes telomere length variation. $(A)$ The frequency of replication fork collapse in wild type, cdc13-F539A and cdc13-F539A exo1- $\Delta$ strains $(8,223,2,836$ and 14,957 colonies, respectively), assessed using the assay described in Fig. 2. (B) An alignment of sequenced telomeres from the progeny of an $\mathrm{Ade}^{-}$half-sector from an est1-60 strain, following replication fork collapse at the interstitial tract. $(C)$ and $(D)$ Sequences of 251 Chr I-L telomeres from the cdc13-F539A strain, aligned based on overall length $(C)$ or the length 
of $\mathrm{G}_{1-3} \mathrm{~T}$ founder sequence DNA $(D)$; representations of the wild type overall length profile (in black, from Fig. $1 B$ ) or the wild type founder length profile (in aqua, from Fig. $1 E$ ) are superimposed on the corresponding cdc13-F539A profiles. The statistical difference between the wild type and $c d c 13-$ F539A profiles was $p<1 \times 10^{-6}$, as determined by a Kolmogorov Smirnov test. Founder sequence DNA is indicated in blue; green corresponds to DNA synthesized by telomerase. $(E)$ The average rate of founder sequence loss for wild type, cdc13-F539A and cdc13-F539A ex01- $\Delta$ strains, calculated as described in Materials and Methods. $(F)$ and $(G)$ The extent of elongation by telomerase at individual telomeres in wild type $(F)$ and cdc13-F539A $(G)$ strains, aligned based on the founder sequence profiles shown Fig $1 E$ and Fig. $4 D$, respectively. $(H)$ The percentage of individual telomeres that were elongated by telomerase by $\geq 150$ nucleotides, for wild type, cdc13-F539A and cdc13-F539A exo1- $\Delta$ strains. (I) Sequences of 189 Chr I-L telomeres from the cdc13-F539A exo1- $\Delta$ strain, aligned based on founder sequence length, with the wild type founder length profile (in aqua, from Fig. $1 E$ ) superimposed. $(J)$ The extent of elongation by telomerase at individual cdc13-F539A exo1- $\Delta$ telomeres, aligned based on the founder sequence profile in $(I)$. $(K)$ Viability of the indicated strains, grown for 4 days at $30^{\circ}$; the colony size of the $c d c 13-\mathrm{F} 539 \mathrm{~A}$ strain increases with additional incubation. $(L)$ The frequency of replication fork collapse, assessed at $23^{\circ}, 30^{\circ}$ and $31^{\circ} \mathrm{C}$, in wild type (5800 to 6400 colonies), cdc13-ts (2300 to 3300 colonies) and cdc13-ts exo1- $\Delta$ (3700 to 3900 colonies) strains; as shown in $(M)$, the semi-permissive temperature for this $c d c 13$-ts strain $(57)$ was $31^{\circ} \mathrm{C}$. $(M)$ Serial dilutions of isogenic strains with the indicated genotypes were plated on pre-warmed plates and incubated at $30^{\circ}, 31^{\circ}, 32^{\circ}$ and $33^{\circ} \mathrm{C}$ for 2 days. 
A

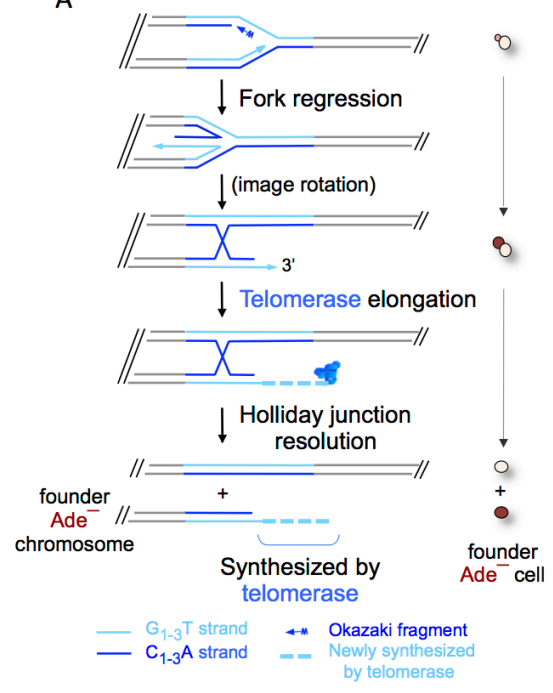

B

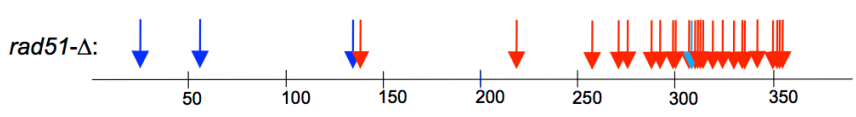

C

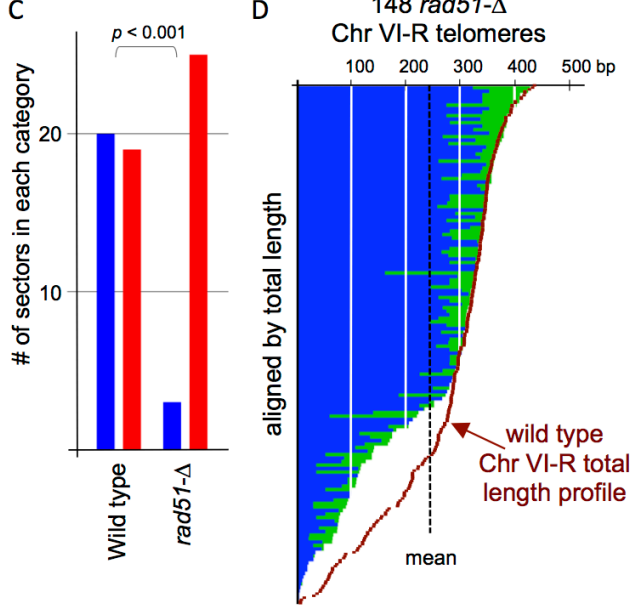

E

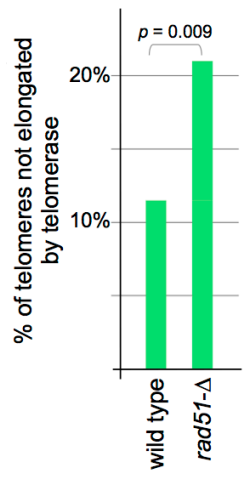

Figure 5. Telomerase-mediated elongation of collapsed forks is reduced in a rad51- $\Delta$ strain.

(A) Illustration showing regression of a stalled replication fork during duplex telomeric DNA replication to produce a 3' G-rich single-strand extension. If this substrate is elongated by telomerase (i.e. in the same cell division), progeny in the resulting $\mathrm{Ade}^{-}{ }^{-}$sector will descend from a single telomerase-elongated founder chromosome, with a pattern of inheritance as observed in examples shown in Fig. 3 and SI Appendix, Fig. S7. Elongation of telomerase is indicated by a dotted line; for clarity, subsequent synthesis of the corresponding C-strand is omitted. (B) A map of the $390 \mathrm{bp}$ interstitial telomeric tract, showing the position at which telomerase initiated synthesis in 28 Ade $^{-}$half-sectored colonies in a rad51- $\Delta$ strain; blue and red arrows as in Fig. $3 C$. The extent of telomerase-mediated elongation in the $\mathrm{Ade}^{-}$founder cell division for the three rad51- $\Delta$ Ade $^{-}$half-sectors indicated by blue arrows is shown in SI Appendix, Fig. S8. (C) The frequency of sectors categorized as either "blue" or "red" for wild type and rad51- $\Delta$ strains, as depicted in the maps in Fig. $3 C$ and Fig. $5 B$, respectively; light blue sectors (2 for wild type and 1 for rad51- $\Delta$ ), as defined in the Fig. $3 C$ legend, are not included. (D) Chr VI-R telomere length profile (148 isolates) from the rad51- $\Delta$ strain, aligned based on overall length; the mean length was $246 \mathrm{bp}$ (9\% shorter than the wild type Chr VI-R mean length). The statistical difference between the wild type and rad51- $\Delta$ profiles was $p=0.00013$, based on a Kolmogorov Smirnov test. $(E)$ The percentage of wild type vs. rad51- $\Delta$ Chr VI-R telomeres that failed to be elongated by telomerase. 